Article

\title{
Structural Holes in Social Networks with Exogenous Cliques
}

\author{
Antoni Rubí-Barceló \\ Departament d'Economia Aplicada, Universitat de les Illes Balears, Campus UIB, \\ 07122 Palma de Mallorca, Spain; antoni.rubi@uib.eu
}

Received: 12 June 2017; Accepted: 25 July 2017; Published: 1 August 2017

\begin{abstract}
It has been empirically shown that structural holes in social networks enable potential large benefits to those individuals who bridge them (Burt, 2004). The work in Goyal and Vega-Redondo (2007) shows that the large payoff differentials caused by structural holes can persist even when agents strategically add and remove ties to smooth those differentials, thereby providing a game-theoretic rationale for the existence of bridge-agents. The present paper ties back to the initial empirical literature by explicitly assuming that agents are exogenously linked forming cliques, as in a firm environment. In this setting, bridge-agents cannot be sustained under the same conditions of Goyal and Vega-Redondo (2007). Instead, they can be sustained when the deviation possibilities are restricted and only when they connect small groups of agents to the rest.
\end{abstract}

Keywords: network formation; structural holes; intermediation; firm organization

\section{Introduction}

Social networks provide a platform for the flow of information because social connections supply individuals with novel ideas, trade opportunities or job vacancies. This suggests that individuals payoffs are highly dependent on their position in the social network.

An important line of work in social network research analyzes this dependence and argues that individuals benefit when they serve as intermediaries or "bridges" between otherwise unconnected groups. The reasons for the success of bridge-individuals have been advanced by Burt's theory of structural holes: bridge-agents can control the flow of information between different groups in the network, adapting it to specific strategic interests. Moreover, they are well-positioned to synthesize ideas coming from different groups, and this enhances their creative capacity [1]. The works in [2-4] provide empirical evidence that people who bridge structural holes in social networks have significantly higher payoffs. From this perspective, there is a natural strategic aspect when selecting personal and business contacts, especially in settings where brokerage and information access and control incentives can reasonably be expected to dominate behavior with respect to tie formation, as in a firm environment.

The analysis in [1] constitutes a basic empirical background of the theoretical analysis developed here. Burt's paper focused on data describing 673 managers who ran the supply chain in 2001 for one of America's largest electronic companies. The study shows that there are clusters of managers within business units. To make the clusters more apparent, Burt looked at the top 89 senior people to see the core of the supply-chain network, and this provided a stark illustration of the fragile contact across business units. Burt's results show that compensation, positive performance evaluations, promotions and good ideas are disproportionately in the hands of managers bridging structural holes.

The work in [5] speculated that this situation could not be sustained in equilibrium if more and more people strategically add and remove ties to gain intermediation rents and to circumvent others who are trying to become intermediary. The work in [6] formally confirmed that speculation in a 
setting where agents try to minimize their network constraint, a measure of structural disadvantage developed in [7]. On the contrary, economic theory has demonstrated that social structures can equilibrate toward highly asymmetric networks with high differential payoffs, even when all agents have complete information and perfectly identical preferences and abilities to strategically modify the network. For example, [8] or [9] identify, as a prominent equilibrium network, the "star", a structure in which a unique agent brokers everyone else. The analysis in [10] also shows in a symmetric setting that individuals can differentiate and receive very different payoffs in equilibrium. All of these papers provide a game-theoretic rationale to contradict Burt's speculation about the impossibility to sustain structural advantages in the long run.

In the model presented here, agents are assumed to be exogenously grouped into clusters, replicating the departments of an organization; namely a firm, a university, a family or a criminal group, among others. This connects this paper to [11], in which a network is endogenously built onto a pre-existing structure, or to [12], where agents are part of a coalition structure apart from being connected to others through a network. The members of a cluster are assumed to be fully linked among them, forming a clique, a notion widely used in network theory. These cliques have possibly different sizes. Agents face the trade-off between the benefits from connecting to other departments' members and the cost, in the effort, to maintain those links. These benefits are determined by the payoff function introduced by [9]: every pair of (directly or indirectly) linked agents creates a unit of surplus that is evenly split across the two involved agents and, possibly, the intermediaries. Thus, individuals form links with other departments' members to create surplus, to gain intermediation rents and to circumvent others who are trying to become intermediary. Unlike [10], links can only be created by mutual consent of the two involved agents.

The main results of this paper show that, in this framework, bridge-agents cannot be sustained in equilibrium under the same conditions of [9]. This is because having groups of completely connected agents multiplies the possibilities of circumventing bridge-agents among different departments. In the setting of the present paper, allowing the same deviation possibilities of [9] is shown to be incompatible with the existence of bridge-agents in equilibrium. However, if possible deviations are restricted, bridge-agents can be sustained, but only if they connect sufficiently small parts of the population to the rest. In this case, bridge-agents can obtain large payoff differentials in equilibrium as illustrated by the examples of Section 3. These results formalize Burt's conjectures about the impossibility to sustain bridge-agents in a firm environment: they cannot connect two big parts of the firm and cannot exist under wide deviation possibilities. Results might also be interpreted as a departure from the sharp results in [9] because they contribute specific conditions to sustain bridge-managers in the equilibrium network of connections of a population.

\section{The Model}

The basic setting is borrowed from [9], except for the group structure of the population. Let $N=\{1,2, \ldots, n\}$ be the finite set of individuals. These individuals are exogenously distributed across different groups or departments. Let $M=\{1,2, \ldots, m\}$ be the finite set of departments of the population, where $m \geq 3$. Each individual is located at exactly one department. Let $M_{i}$ be the department of individual $i$, and let $m_{i}=\left|M_{i}\right|$ denote the size of this department. Let $m_{i} \geq 2, \forall i \in N$.

Agents are connected by a graph or network $g$, a collection of direct links that represent pairwise relations between the respective two individuals, with the particularity that all members of the same department are fixedly connected to each other, forming a clique. The set of all of these possible networks in $N$ is $G$. The subset of $N$ containing two individuals $i$ and $j$ is denoted by $i j$ and is referred to as the link $i j$. Individuals $i$ and $j$ are connected if and only if $i j \in g$. Thus, if $j \in M_{i}$, then $i j \in g$, by assumption. When $i$ and $j$ belong to different departments, then link $i j$ is said to be external. A path in $g$ connecting $i_{1}$ and $i_{t}$ is a set of distinct nodes $\left\{i_{1}, i_{2}, \ldots, i_{t}\right\} \subset N$ such that $\left\{i_{1} i_{2}, i_{2} i_{3}, \ldots, i_{t-1} i_{t}\right\} \subset g$. All individuals with whom $i$ has a path constitute the component of $i$ in $g$, which is denoted by $C_{i}(g)$. 
Two departments $M_{1}$ and $M_{2}$ are connected if and only if there is a pair of individuals $i \in M_{1}$ and $j \in M_{2}$ such that $i j \in g$.

Individuals play a network-formation game where the strategy of a player consists of making an announcement of intended external links. Let $s_{i}$ be the strategy vector of player $i$, which has $n-m_{i}$ elements. Let $s_{i j} \in\{0,1\}$ be a particular element of this vector, where $s_{i j}=1$ means that player $i \in M_{i}$ intends to form a link with player $j \in M_{j}$ (where $M_{i} \neq M_{j}$ ), while $s_{i j}=0$ means the opposite. A link between two individuals is undirected, can be severed by one of them unilaterally, but can only be created by mutual consent of the two implied individuals. Formally, a link between $i$ and $j$ is formed if and only if $s_{i j} s_{j i}=1$. Notice that a strategy profile $s=\left(s_{1}, s_{2}, \ldots, s_{n}\right)$ induces a unique network $g(s)$.

As in [9], the payoff function is such that any pair of connected players ( $i$ and $j$ ) generates one unit of surplus. The distribution of this unit depends on the intermediaries between $i$ and $j$ and on the nature of competition between intermediaries. It is assumed that any two paths between any two players fully compete away the entire surplus (à la Bertrand competition). Therefore, an intermediary between $i$ and $j$ (say $k$ ) can retain part of the surplus generated by $i$ and $j$ if and only if this intermediary lies on all paths connecting $i$ and $j$. In such a case, $k$ is said to be an essential agent for $i$ and $j$. For example, in a star network where a unique agent $i$ is connected to any other agent, whereas others do not hold any additional link, agent $i$ is essential since no pair of players can ever avoid her/him on any path connecting them. Throughout the paper, essential players will also be called bridge-agents. Let $c$ be the cost of an external link. Let $E(j, k ; g)$ be the set of essential agents in $g$ between $j$ and $k$, and let $e(j, k ; g)=|E(j, k ; g)|$. Then, for every strategy profile $s$, net payoffs to player $i$ are given by:

$$
\Pi_{i}(s)=\sum_{j \in C_{i}(g)} \frac{1}{e(i, j ; g)+2}+\sum_{j, k \in N} \frac{I_{\{i \in E(j, k)\}}}{e(j, k ; g)+2}-\eta_{i}(g) c
$$

where $I_{\{i \in E(j, k)\}}$ is an indicator function specifying whether $i$ is essential for $j$ and $k$ and $\eta_{i}(g) \equiv \mid\{j \in$ $\left.N: j \notin M_{i}, i j \in g\right\} \mid$ denotes the number of external links of $i$. The first term represents $i$ 's access payoffs while the second term represents her/his intermediation payoffs. A network $\tilde{g}$ is efficient if $W(\tilde{g}) \geq W(g)$ for all $g \in G$, where $W(g) \equiv \sum_{i \in N} \Pi_{i}(s)$ and $g=g(s)$. Finally,

Definition 1. Agent $x \in C_{x}(g)$ is central if and only if:

$$
x \in \underset{i \in C_{x}(g)}{\arg \max } \sum_{j \in C_{x}(g)} \frac{1}{e(i, j ; g)+2} .
$$

In other words, $x$ is central if no member of $C_{x}(g)$ receives more access payoffs than $x$. For example, in a star network, the agent connected to all others is the unique central agent of the network. On the other hand, in a cycle network where all agents are connected to two other agents forming a circle, all agents are central.

At this point, I present some graph-theoretic notions that will be used repeatedly throughout the paper. A link is said to be critical if it defines the unique path between the two players involved and whose deletion increases the number of components. If all individuals belong to the same component, the population is said to be connected. An isolated department does not have external links. A network without external links is said to be empty. If a component $C_{i}(g)$ contains an essential player $i$, then the rest of the members of $C_{i}(g)$ can be distributed among two or more $i$-groups; $j, k \in C_{i}(g)$ are members of different $i$-groups if $i$ is essential for connecting them. A department $M_{i}$ is essential if there is a pair of departments $M_{j}$ and $M_{k}$ such that every path that links some member of $M_{j}$ to some member of $M_{k}$ contains a member of $M_{i}$ (not necessarily the same). Notice that essential agents can be members of both essential and non-essential departments. A non-essential department can be extreme or not. Department $M_{i}$ is extreme if it is only connected to another department. Otherwise, $M_{i}$ is said to be non-extreme. If a component $C_{i}(g)$ contains an essential department $M_{i}$, then the rest of the 
departments of $C_{i}(g)$ can be distributed among two or more $M_{i}$-groups: $j, k \in C_{i}(g)$ are members of different $M_{i}$-groups if all paths connecting $j$ and $k$ include some member of $M_{i}$. Finally, a group of $p$ departments constitutes a cycle if they can be ordered in a list $M_{1}, M_{2}, \ldots, M_{p}$ such that $M_{p}$ is connected to $M_{1}$ and $M_{i}$ is connected to $M_{i+1}$ for $i=\{1,2, \ldots, p-1\}$ and there are no other external links.

\section{Equilibrium Concept}

Given that link creation requires mutual consent of the two players involved and that agents can announce any combination of links they wish (multidimensional strategy space), a coordination problem arises. As such, the game displays a multiplicity of Nash equilibria where mutually-beneficial links can be left aside. ${ }^{1}$ This is solved if players are allowed to coordinate bilaterally. For this reason, refinements on the Nash equilibrium that allow for coalitional moves are usually applied to this kind of network formation game. One of the most widely-used refinements is the pairwise-Nash equilibrium, created by [13], which is defined as follows:

Definition 2. A strategy profile $s^{P N}$ is a Pairwise-Nash Equilibrium (PNE) if the following conditions hold:

(i) for any $i \in N$ and every $s_{i} \in S_{i}, \Pi_{i}\left(s^{P N}\right) \geq \Pi_{i}\left(s_{i}, s_{-i}^{P N}\right)$

(ii) for any pair of players $i, j \in N$ and every strategy pair $\left(s_{i}, s_{j}\right)$ in which $s_{i l}=s_{i l}^{P N}, \forall l \neq j$ and $s_{j k}=$ $s_{j k}^{P N}, \forall k \neq i$,

$$
\Pi_{i}\left(s_{i}, s_{j}, s_{-i-j}^{P N}\right)>\Pi_{i}\left(s_{i}^{P N}, s_{j}^{P N}, s_{-i-j}^{P N}\right) \Rightarrow \Pi_{j}\left(s_{i}, s_{j}, s_{-i-j}^{P N}\right)<\Pi_{j}\left(s_{i}^{P N}, s_{j}^{P N}, s_{-i-j}^{P N}\right) .
$$

Networks generated by a PNE strategy profile $g\left(s^{P N}\right)$ are robust to deviations of unilateral multilink severance (that is, the usual Nash Equilibrium requirement) and to deviations of bilateral commonly-agreed one-link creation. That is, a PNE network is a Nash equilibrium network where, in addition, no mutually-beneficial link can be formed.

Alternative equilibrium notions include unilateral stability concepts as in [6] or [10] and concepts that allow for other coalitional moves. ${ }^{2}$ The Bilateral Equilibrium (BE) concept used in [9] deserves special attention. The bilateral equilibrium concept is defined as follows:

Definition 3. A strategy profile $s^{B}$ is a Bilateral Equilibrium (BE) if the following conditions hold:

(i) for any $i \in N$ and every $s_{i} \in S_{i}, \Pi_{i}\left(s^{B}\right) \geq \Pi_{i}\left(s_{i}, s_{-i}^{B}\right)$

(ii) for any pair of players $i, j \in N$ and every strategy pair $\left(s_{i}, s_{j}\right)$,

$$
\Pi_{i}\left(s_{i}, s_{j}, s_{-i-j}^{B}\right)>\Pi_{i}\left(s_{i}^{B}, s_{j}^{B}, s_{-i-j}^{B}\right) \Rightarrow \Pi_{j}\left(s_{i}, s_{j}, s_{-i-j}^{B}\right)<\Pi_{j}\left(s_{i}^{B}, s_{j}^{B}, s_{-i-j}^{B}\right) .
$$

A BE network must be robust to bilateral commonly-agreed one-link creation, to unilateral multilink severance and to deviations consisting of a simultaneous combination of the previous two deviations by any given pair of individuals. In particular, Condition (ii) implies that a BE network should be robust to any possible coalitional deviation that involves two agents. Thus, under the BE concept, agents can use more complex deviations than under the PNE concept. Proposition 1 shows that, unlike [9], bridge-agents cannot be sustained in a BE network in the present setting. However, if the deviation possibilities are restricted, so that either only one individual considers deleting her/his links or two individuals consider creating a link between them at a time, equilibrium networks can display bridge-agents enjoying large payoff differentials.

1 For example, a strategy profile in which no player announces a link (resulting in the empty network) is always a Nash equilibrium.

2 See [14] and [15]. 


\section{Results}

First, the set of efficient networks is characterized. It is trivial to see that a network is efficient if and only if it is empty or minimally connected, i.e., all departments are grouped into the same component, and there are $m-1$ external links. As a consequence, notice that in the present setting, all agents with external links in an efficient network are essential. Therefore, efficient networks other than the empty network display multiple essential agents.

Now, the study turns to the equilibrium analysis. The following two results narrow the set of PNE networks and, since BE is stricter than PNE, also the set of BE networks. All proofs are relegated to the Appendix A.

Lemma 1. A PNE network $g^{*}$ cannot include more than one multi-department component $\hat{C}_{i}\left(g^{*}\right)$. Moreover, an isolated department $M_{j}$ can coexist with $\hat{C}_{i}\left(g^{*}\right)$ only if:

(i) non-essential agents do not have external links and

(ii)

$$
c>\frac{1}{2}+\frac{m_{j}-1}{3}+\sum_{l \in C_{i}(g)} \frac{1}{e(i, l ; g)+3}+\left(m_{j}-1\right) \sum_{l \in C_{i}(g)} \frac{1}{e(i, l ; g)+4},
$$

where $i$ is a central member of $\hat{C}_{i}\left(g^{*}\right)$.

The proof shows that the marginal access and intermediation payoffs for those who create a critical link between two multi-department components always exceed the payoffs of (at least) one member of these components, so if this member does not have incentives to cut all of his/her links off, then creating a critical link should be a profitable deviation. Similarly, the proof of Part (i) shows that the marginal gross intermediation and access payoffs obtained by a pair of agents who create a critical link between them exceed the actual gross payoff of a non-essential agent with some external link, so if the linking cost is sufficiently low to sustain this non-essential agent in equilibrium, then a pair of members of different components will have incentives to create a link between them. Finally, Part (ii) states that in order to sustain two separate components in equilibrium, the linking cost should exceed the gross marginal payoff of the most profitable creation of a critical link between them. This result implies that there can be three different equilibria: (a) a connected network; (b) an empty network or (c) a network with a unique multi-department component and sufficiently small isolated departments. ${ }^{3}$ The last equilibrium is inefficient, whereas the last two equilibria reflect a coordination problem and can only be sustained for sufficiently high values of $c .^{4}$

In order to analyze the conditions under which bridge-agents can be sustained in equilibrium, the analysis should focus on the unique multi-department component.

Lemma 2. Given a sufficiently large multi-department component, ${ }^{5}$ a PNE network cannot display more than one essential agent for any $c$.

If two agents create a link circumventing (at least) one essential agent, then each of them will eliminate (at least) one intermediary to access a specific part of the population. Thus, only when the size of some of these two parts is lower than an upper bound (that depends on $c$ ), this link will not be formed. The proof shows that if there are two or more essential agents in the same component,

Condition (ii) of Lemma 1 specifies the upper-bound of the size of isolated departments as a function of $c$.

4 For example, the empty network is a PNE if $c>\frac{1}{2}+\frac{1}{3}\left(m_{j}-1\right)+\frac{1}{3}\left(m_{i}-1\right)+\frac{1}{4}\left(m_{j}-1\right)\left(m_{i}-1\right)$ for any pair of isolated departments $M_{i}$ and $M_{j}$.

5 In the spirit of Goyal and Vega-Redondo (2007) (see their Footnote 10), this requirement can be stated more precisely by saying that there is a function $F(c)$ such that if the size of the multi-department component exceeds $F(c)$, this result is obtained. This is the interpretation of most of the results in this paper. The particular form of the lower-bound $F$ would depend on the result under consideration, and its specification is beyond the scope of that paper. 
then this upper bound will be exceeded for some pair of agents; so, there always exist two individuals who can profitably circumvent some essential agent. Furthermore, a unique essential agent can be sustained in a large multi-department component and only if he/she connects sufficiently small parts of the component to the rest.

Since a multi-department component with (at most) one essential agent cannot be minimally connected, the previous result implies that if the population is large, then PNE (and also BE) networks will not be efficient. ${ }^{6}$ Moreover, the previous lemma implies that some non-essential agent will hold external links in this multi-department component. Thus, from Lemma 1, the next directly follows:

Corollary 1. Given a sufficiently large multi-department component, a PNE network should be connected for any $c$.

As commented above, this list of necessary conditions stated by Lemmas 1 and 2 restricts both the set of potential PNE and BE networks. Based on these results, the next proposition follows:

Proposition 1. Given a sufficiently large multi-department component, a BE network cannot include any essential agent.

From Lemma 2, a PNE network cannot have more than one essential agent. This will also be true for BE networks. The proof focuses on this unique essential agent to show that, under the BE concept, agents have the deviation possibilities they need to circumvent it. In particular, a pair of deviators is allowed to coordinate the replacement of a set of their links with a new link between them. In our setting, this allows deviators to circumvent essential agents without any extra cost. Consequently, bridge-agents cannot be sustained in BE networks.

This result contrasts with [9] where the unique (strict) BE network displays one essential agent. The existence of multi-personal nodes, as the departments of our setting, has a double effect: on the one hand, this potentially increases the benefits from holding links to other nodes, but on the other hand, this may also smooth payoff differentials because there are more possibilities to circumvent bridge-agents. For this reason, only when these possibilities are restricted with respect to [9], by using a weaker equilibrium concept as is done next, large payoff differentials can be sustained in equilibrium.

In spite of the list of necessary conditions stated by Lemmas 1 and 2, the set on PNE networks is large. However, the next result shows that under certain network structures, bridge-agents must necessarily exist in equilibrium.

Proposition 2. Given a sufficiently large multi-department component with an essential department $M_{j}$ and an $M_{j}$-group containing $t$ agents, there exists a $\tilde{c}(t) \geq \frac{3 t-1}{12}$ such that, for any $c>\tilde{c}(t), a$ PNE network must display a unique essential agent $j \in M_{j}$ who connects those $t$ agents to the rest of the component.

In other words, if an essential department $M_{j}$ has an $M_{j}$-group relatively small with respect to $c$ in a sufficiently large component, then a unique agent $j$ will be essential for connecting the members of this $M_{j}$-group to the rest of the component. The proof shows that $j$ must have two external links to this $M_{j}$-group, say $j k$ and $j h$, and that there is no other way to connect this $M_{j}$-group to $M_{j}$. Intuitively, there are several factors that explain this result. First, agent $k$ (or $h$ ) does not have incentives to cut $j k$ (or $j h$ ) off because this would involve an additional essential agent to access the rest of the component, which is sufficiently large to make this deviation nonprofitable. Similarly, agent $j$ does not have incentives to cut some of her/his external links off because this would imply a loss of intermediation payoffs that exceeds the cost saving. Finally, no other agent has incentives to circumvent agent $j$ to access this $M_{j}$-group because this group is too small to compensate the cost of a new link. Thus, the smaller the 
$M_{j}$-group, the smaller will be $\tilde{c}(t)$, so the essential department $M_{j}$ will have to include one essential agent for a wider range of $c$.

In general, a multi-department component without essential agents can present multiple structures in equilibrium. However, the previous result narrows the possibilities: a multi-department component without essential agents can be sustained when (1) it does not include essential departments, i.e., this component is a cycle or a group of cycles; or (2) all $M_{i}$-groups are sufficiently large, for any essential department $M_{i}$ in the component.

The next result follows from Proposition 2 and Lemma 2.

Corollary 2. Given a sufficiently large multi-department component, a PNE network can only include one essential department $M_{j}$ with some $M_{j}$-group sufficiently small. ${ }^{7}$ Moreover, if there are multiple $M_{j}$-groups sufficiently small, then they must be connected to $M_{j}$ through the same essential agent, $j \in M_{j}$.

Moreover, a sufficiently small $M_{j}$-group cannot include an essential department itself because, by Proposition 2, this would imply a new essential agent, which contradicts Lemma 2.

The following example illustrates a PNE network with one essential department.

Example 1. Consider a connected network with a unique essential player $i$ who has two external links to every other department. Moreover, there are no additional links. Let $m$ be the number of departments. For simplicity, it is assumed that all departments have the same size s. I claim that such a network is a PNE if $m$ is sufficiently large and:

$$
c \in\left(\frac{s}{6}, \frac{s}{12}\left[s(m-2)+2(s-1)+3 \frac{s m-1}{s(m-1)}\right]\right),
$$

so that $c$ is sufficiently high to avoid an additional link and sufficiently low to avoid the deletion of an external link.

In this case, player i's payoffs are positive and equal to:

$$
\frac{s^{2}(m-1)(m-2)}{6}+\frac{s m-1}{2}+\frac{(s-1) s(m-1)}{3}-2(m-1) c
$$

Player i's marginal payoff from cutting one link off is:

$$
c-\frac{s-1}{6}-\frac{(s m-s+2)(s-1)}{12}
$$

which is negative for a sufficiently high $m$. Likewise, it can be concluded that i's marginal payoff for cutting two links to a department is also negative. On the other hand, if a player in a peripheral department deletes one external link, then she/he obtains a marginal payoff equal to:

$$
c-\frac{1}{6}-\frac{s m-s-1}{12}
$$

which is negative for sufficiently large $m$. The creation of an additional link generates the following marginal payoff for one of the deviators:

$$
\frac{s}{6}-c
$$

which is negative given the conditions stated in this example. Therefore, it can be concluded that this network is PNE under those conditions of $m$ and $c$.

Moreover, by Proposition 2, this is the unique form of connecting all of the peripheral departments to the essential one when $c>\frac{3 s-1}{12}$.

\footnotetext{
$7 \quad$ An $M_{j}$-group with $t$ agents is sufficiently small if $c>\tilde{c}(t)$.
} 
Notice that for moderate values of $c$, agent $i$ will enjoy large payoff differentials with respect to others because of the large intermediation payoffs she/he receives.

The previous example illustrates that bridge-agents not only can be sustained in PNE networks, but also that they must exist, under certain configurations of the network. Moreover, the example shows that these agents can enjoy much larger payoffs than others in equilibrium.

Essential agents can also be members of non-essential departments if the following necessary conditions hold.

Proposition 3. An extreme department $M_{j}$ can contain an essential agent only if $m_{j} \in\{2,3\}$. A PNE network can display an essential agent in a non-essential department $M_{i}$ only if $c>\frac{m_{i}-1}{6}$.

Therefore, an essential agent can monopolize the intermediation between a group of agents and the rest of the component only if this group is sufficiently small. The previous result establishes specific size upper-bounds for non-essential departments. Below these upper-bounds, no other agent has incentives to circumvent the essential agent to access such a small group because the additional access payoffs do not compensate the cost of the new link. Nevertheless, the essential agent enjoys large payoff differentials with respect to the rest of the agents, as the next example illustrates.

Example 2. Consider a network consisting of a cycle of $m$ departments. Only one of them, say $M_{i}$, has a single player $i$ with external links. All other departments have at least two players with external links. In that case, agent $i$ is essential for connecting the rest of the members of her/his department to the rest of the component. This network is a PNE if $m$ is sufficiently large and $m_{i}<6 c+1$.

For any given $c$, it is easy to see that the marginal payoff for deleting one external link will be negative for a sufficiently large $m$. On the other hand, the most profitable possibility for creating a new link, i.e., adding a link circumventing the essential player $i$, generates a marginal payoff to one of the deviators equal to:

$$
\frac{m_{i}-1}{6}-c
$$

which is negative under the initial conditions stated above. Thus, the network is a PNE. Notice that the payoff of $i$ is:

$$
\Pi_{i}=\frac{n-1}{2}+\frac{\left(n-m_{i}\right)\left(m_{i}-1\right)}{3}-2 c
$$

whereas the payoff of any agent not in $M_{i}$ without external links, say $j$, is:

$$
\Pi_{j}=\frac{n-m_{i}}{2}+\frac{m_{i}-1}{3} .
$$

Therefore, for moderate values of $c$, the payoff differential between these two players is high.

\section{Conclusions}

Empirical evidence shows that structural holes in social networks generate potentially large benefits for those individuals who succeed in bridging them and, consequently, large payoff differences among agents. The persistence of such payoff differentials in strategic settings where self-interested agents can alter the network structure could be interpreted as an odd and counterintuitive empirical fact: when brokerage incentives dominate behavior with respect to link formation, one would expect that disfavored agents would strategically add and remove ties to smooth those differentials, as Ronald Burt speculated.

This paper focuses on a setting that can be interpreted as a firm environment, where the aforementioned agents' incentives can reasonably be expected to play a predominant role, to demonstrate that structural advantages cannot be sustained in equilibrium unless the coordination possibilities among 
potential deviators are restricted with respect to [9], which used the (strict) BE concept. This is because the specific configuration of the population (agents are distributed forming exogenously fixed cliques) increases the possibilities of circumventing essential players. Nevertheless, the present paper shows that large payoff differentials can be sustained in PNE networks, that is when agents are not allowed to add and remove links simultaneously; however, only when they broker the connections of a sufficiently small group of agents to the rest. This does not prevent individuals bridging structural holes from enjoying large payoff differentials.

These results contribute some new insights to the sharp results of [9]. Under the interpretation of a firm environment, the relative location of a department in the network of connections might play an important role to explain whether this department can contain essential agents or not; in this respect, an essential agent bridging two big parts of the firm might represent too tempting deviation possibilities for a pair of agents that can circumvent this essential. Likewise, the coordination capabilities of potential deviators are determinant to sustain bridge-agents; in our setting, those capabilities need to be restricted with respect to [9] in order to sustain essential agents. Further empirical research could aim at testing the relevance of these conditions for real social networks to display bridge-agents in the long run.

Acknowledgments: Two previous versions of this paper were entitled "Structural holes and densely connected communities" and "Structural holes in a firm environment". I am grateful to Antonio Cabrales for very helpful comments and suggestions. I have also benefited from comments by Coralio Ballester, Antoni Calvó-Armengol, Joan de Martí, Sanjeev Goyal, Tomás Lejarraga, Fernando Vega-Redondo, Marco van der Leij, Adrien Vigier and from the presentations at XXII Jornadas de Economía Industrial (Barcelona), University of Cambridge and at Libon Meetings in Game Theory and Applications. Financial support from Fundación Ramón Areces and the Spanish Ministerio de Economia y Competitividad and FEDER through Grant ECO2015-67901-P (MINECO/FEDER) is gratefully acknowledged. All errors are of course mine.

Conflicts of Interest: The author declares no conflict of interest.

\section{Appendix A. Proofs}

First, two preliminary lemmas are introduced. Their proof is omitted here because they are immediate applications of two analogous lemmas set forth by [9]. Lemma A1 refers to the marginal payoff of critical links. Notice that critical links can only connect players from different departments; therefore, critical links are not just the unique path between two players, but they are also the unique path between departments.

Lemma A1. Consider any network $g$. If $i j \in g$ and the link is critical, then the marginal payoff of this link for both players ( $i$ and $j$ ) is exactly the same.

Lemma A2. In a network $g$, any component has at least two non-essential departments.

Let $\mathcal{N}_{i}^{r}(g)=\left\{l \in C_{i}(g): e(i, l)=r\right\}$ be the set of agents whom $i$ accesses via $r$ essential players and $\eta_{i}^{r}(g)=\left|\mathcal{N}_{i}^{r}(g)\right|$. Let $R_{i}(g)$ be the maximal number of essential players between $i$ and any other member of her component.

Proof of Lemma 1. Let $g$ be a PNE network, and let $C_{i}(g)$ be a multi-department component. Consider that $g$ includes an additional component $C_{j}(g)$. By contradiction with Part (i), assume that $i \in C_{i}(g)$ is a non-essential agent with at least one external link. In a PNE network, agent $i$ 's payoff should hold:

$$
\Pi_{i} \geq \sum_{r=0}^{R_{i}(g)} \frac{\eta_{i}^{r}(g)}{r+2}-c \geq 0
$$

Let $j \in M_{j}$ be a member of $C_{j}(g)$. Consider the deviation consisting of forming the critical link $i j$. Agent $j$ 's marginal payoff holds: 


$$
\begin{aligned}
\Delta \Pi_{j} & \geq \frac{1}{2}+\sum_{r=0}^{R_{i}(g)} \frac{1}{r+3}+\left(m_{j}-1\right)\left[\frac{1}{3}+\sum_{r=0}^{R_{i}(g)} \frac{1}{r+4}\right]-c \\
& =\frac{1}{2}+\frac{m_{j}-1}{3}+\sum_{r=0}^{R_{i}(g)} \eta_{i}^{r}(g)\left(\frac{1}{r+3}+\frac{m_{j}-1}{r+4}\right)-c \\
& >\frac{1}{2}+\sum_{r=0}^{R_{i}(g)} \eta_{i}^{r}(g)\left(\frac{1}{r+3}+\frac{1}{r+4}\right)-c \\
& \geq \frac{1}{2}+\sum_{r=0}^{R_{i}(g)} \eta_{i}^{r}(g)\left(\frac{1}{r+3}+\frac{1}{r+4}-\frac{1}{r+2}\right)
\end{aligned}
$$

where the last inequality follows from condition $(*)$. Since $\frac{1}{T}+\frac{1}{T+1}-\frac{1}{T-1}>0, \forall T \geq 3$, it can be concluded that $\Delta \Pi_{j}>0$. By Lemma A1, player $i$ will also have incentives to deviate. Thus, $g$ is not a PNE network, contradicting the initial statement and concluding the proof of Part (i).

Next, it is shown that two multi-department components, $C_{i}(g)$ and $C_{j}(g)$, cannot coexist in a PNE network $g$. By Lemma A2, any multi-department component includes two or more non-essential departments. As a consequence, the following two cases exhaust all of the possible configurations of $C_{i}(g)$ :

(a) Some non-essential department in $C_{i}(g)$ (say $M_{i}$ ) is extreme.

Part (i) of Lemma 1 implies that $M_{i}$ can only have one external link (say $i k$ ) in a PNE network. This link should be profitable for both $i$ and $k$. Therefore, creating a new critical link $j k$ bridging $C_{i}(g)$ and $C_{j}(g)$ would be (weakly) profitable for both $j$ and $k$ whenever $m_{j} \geq m_{i}$. Thus, $M_{j}$ should be smaller than the extreme department $M_{i}$ in any PNE network.

(b) All non-essential departments in $C_{i}(g)$ are non-extreme.

Let $M_{i}$ be a non-essential department in $C_{i}(g)$. Notice that Part (i) of Lemma 1 implies that only one agent (say $i$ ) in $M_{i}$ can have external links. Since $M_{i}$ is non-extreme, player $i$ has two or more external links, i.e., $\eta_{i}(g) \geq 2$. Player $i$ 's payoff can be written as:

$$
\Pi_{i}=\sum_{r=0}^{R_{i}(g)} \frac{\eta_{i}^{r}(g)}{r+2}+\left(m_{i}-1\right)\left(\frac{\eta_{i}^{0}(g)-\left(m_{i}-1\right)}{3}+\sum_{r=1}^{R_{i}(g)} \frac{\eta_{i}^{r}(g)}{r+3}\right)-\eta_{i}(g) c
$$

Since $g$ is a PNE network, it follows that:

$$
\frac{1}{\eta_{i}(g)}\left[\sum_{r=0}^{R_{i}(g)} \frac{\eta_{i}^{r}(g)}{r+2}+\left(m_{i}-1\right)\left(\frac{\eta_{i}^{0}(g)-\left(m_{i}-1\right)}{3}+\sum_{r=1}^{R_{i}(g)} \frac{\eta_{i}^{r}(g)}{r+3}\right)\right] \geq c
$$

Agent $j$ 's marginal payoff for forming a new critical link $i j$ between $C_{i}(g)$ and $C_{j}(g)$ is:

$$
\begin{aligned}
\Delta \Pi_{j} & >\frac{1}{2}+\sum_{r=0}^{R_{i}(g)} \frac{\eta_{i}^{r}(g)}{r+3}+\left(m_{j}-1\right)\left(\frac{1}{3}+\sum_{r=0}^{R_{i}(g)} \frac{\eta_{i}^{r}(g)}{r+4}\right)-c \\
& >\sum_{r=0}^{R_{i}(g)} \frac{\eta_{i}^{r}(g)}{2 r+4}+\left(m_{j}-1\right)\left(\sum_{r=0}^{R_{i}(g)} \frac{\eta_{i}^{r}(g)}{2 r+6}\right)-c \\
& \geq \frac{1}{\eta_{i}(g)}\left[\sum_{r=0}^{R_{i}(g)} \frac{\eta_{i}^{r}(g)}{r+2}+\left(m_{j}-1\right) \sum_{r=0}^{R_{i}(g)} \frac{\eta_{i}^{r}(g)}{r+3}\right]-c
\end{aligned}
$$


The first and second inequalities are immediate, while I use $\eta_{i}(g) \geq 2$ in deriving the third inequality. The last expression can be compared with (**) to conclude that $\Delta \Pi_{j}>0$ when $m_{j} \geq m_{i}$. Thus, $M_{j}$ should be smaller than the non-extreme department $M_{i}$ in a PNE network.

Both cases imply that in a PNE network, any non-essential department of $C_{i}(g)$, say $M_{i}$, should be larger than any department of $C_{j}(g)$. Conversely, if $C_{j}(g)$ is a multi-department component, a non-essential department of $C_{j}(g)$ should be larger than any department of $C_{i}(g)$. These two conditions cannot hold simultaneously, so two multi-department components cannot coexist in a PNE network.

Consequently, only isolated departments can coexist with one multi-department component, $C_{i}(g)$. Let $M_{j}$ be an isolated department. The most profitable critical link between $M_{j}$ and $C_{i}(g)$ would connect $j \in M_{j}$ to a central member of $C_{i}(g)$, say $i$. Agent $j$ 's marginal payoff for the creation of that link would be:

$$
\Delta \Pi_{j}=\frac{1}{2}+\frac{m_{j}-1}{3}+\sum_{l \in C_{i}(g)} \frac{1}{e(i, l ; g)+3}+\left(m_{j}-1\right) \sum_{l \in C_{i}(g)} \frac{1}{e(i, l ; g)+4}-c
$$

Condition (ii) of Lemma 1 trivially follows from here.

Proof of Lemma 2. By contradiction, let us assume that there are at least two essential players $i$ and $j$ in a PNE network $g$. Notice that these two players must be located in a multi-department component (say $C_{i}(g)$ ). This component can be divided into three parts: (1) one $i$-group that does not contain $j$, (2) one j-group that does not contain $i$ and (3) the rest of the component. Let $k$ and $l$ be two agents contained in Parts (1) and (2), respectively.

The creation of the link $k l$ will be profitable for a sufficiently large size of Part (3) because $k$ and $l$ will circumvent an essential player ( $i$ and $j$, respectively) to reach this part of the component. On the other hand, if $l$ and $i$ form a link between them, then $i$ increases her/his payoffs obtained from the intermediation between Parts (1) and (2), and $l$ circumvents an essential player from reaching Part (1). This deviation is profitable for a sufficiently large Part (1) because both marginal payoffs are increasing in the size of this part. Similarly, the creation of the link $k j$ will be profitable for a sufficiently large Part (2). By construction, one of these three parts should be sufficiently large in a sufficiently large component, so there always exists a profitable deviation for a sufficiently large $C_{i}(g)$, contradicting the initial statement.

Proof of Proposition 1. By contradiction, it is assumed that a BE network $g$ contains an essential agent $i$. Since BE is stricter than PNE, Lemmas 1 and 2 also hold under the BE concept. Thus, the multi-department component cannot include more than one essential agent $i$. At this point, two different cases need to be distinguished:

(a) Agent $i$ is contained in an essential department $M_{i}$.

Let $M_{j}$ and $M_{k}$ be two departments, such that the essential player $i$ is part of all paths between the members of $M_{j}$ and $M_{k}$. Since there does not exist any additional essential agent, individual $i$ must have two or more links to at least one of the $M_{i}$-groups containing $M_{j}$ or $M_{k}$. Take two agents from $M_{j}$ and $M_{k}$ linked to the essential department $M_{i}$. Notice that at least one of them is linked to $i$. Then, consider the deviation consisting of deleting their links to $M_{i}$ and forming a link between them. ${ }^{8}$ Without increasing their costs, they will circumvent the essential player $i$ to access the other $M_{i}$-group. Therefore, they will strictly increase their access payoff. Therefore, they have incentives to deviate, contradicting the initial statement. 
(b) Player $i$ is contained in a non-essential department $M_{i}$.

There are two types of non-essential departments. Next, I develop them in turn:

(i) $\quad M_{i}$ is an extreme department. Let $i$ be an essential agent in $M_{i}$. Since $i$ is the unique essential agent in $M_{i}$, she/he must have at least two links, say $i k, i j \in g$, where $k, j \in M_{j}$. Consider the deviation in which $j$ severs the link $i j$ and forms a new link $j l$ where $l \in M_{i}$ and $l \neq i$. The marginal payoff for agent $j$ will be positive, given that without increasing her/his linking costs, the essential agent $i$ will be avoided in order to reach the members of $M_{i}$. On the other hand, by forming such a link, agent $l$ would eliminate the essential agent $i$ to reach the rest of the component at the cost of one additional link (c). For a sufficiently large component, agent $l$ would also have incentives to deviate. Consequently, an extreme department cannot include essential agents in a BE network.

(ii) $\quad M_{i}$ is a non-extreme and non-essential department. Notice that $i \in M_{i}$ can be essential if and only if she/he is the unique agent in $M_{i}$ with external links. Let $i j \in g$ where $j \notin M_{i}$. Consider that $j$ severs the link $i j$ and simultaneously forms a link $j k$ where $k \in M_{i}$ and $k \neq i$. With this deviation, $j$ will have two different paths to communicate with any member of $M_{i}$ without any additional cost. Then, the marginal payoff of agent $j$ will be positive since she/he avoids one essential agent. On the other hand, agent $k$ will circumvent the essential agent $i$ to access the rest of the component with an additional cost of $c$. Thus, for a sufficiently large component, $k$ will also deviate. Consequently, this kind of department cannot contain essential agents either.

Proof of Proposition 2. First, let us assume that $g$ is a PNE network that displays a multi-department component with an essential department $M_{j}$. By Lemma 2, in a sufficiently large component, department $M_{j}$ cannot be connected to any $M_{j}$-group through one critical link because this network would display two essential agents. On the other hand, whenever there are more than two links between some $M_{j}$-group and $M_{j}$, some agent will have incentives to deviate by deleting one external link; the deviator will save $c$ without affecting access and intermediation payoffs. Let us analyze the remaining cases in turn.

Let us assume that there are two links involving four different players, say $j k$ and $i h$, where $j, i \in M_{j}$ and $k, h$ are members of the $M_{j}$-group. Let $t$ be the number of members of this $M_{j}$-group. By deleting the link $j k$, agent $j$ obtains $\Delta \Pi_{j}=c-\tilde{c}(t)$. Specifically, $\tilde{c}(t)=\frac{3 t-1}{12}$ when the deletion of the link $j k$ creates two new essential agents, $i$ and $h$. If this deviation creates additional essential agents because of the structure of the $M_{j}$-group, then $\tilde{c}(t)>\frac{3 t-1}{12}$. In any case, the deviation is profitable whenever $c>\tilde{c}(t)$.

Let us assume that there are two links between $M_{j}$ and the $M_{j}$-group, say $j k$ and $i k$, where $j, i \in M_{j}$ and $k$ is an essential agent in the $M_{j}$-group. Trivially, when $c>\tilde{c}(t)$, agent $j$ (or $i$ ) will have incentives to cut her/his external link off.

Consequently, the unique remaining possibility of connection between $M_{j}$ and the $M_{j}$-group involves two external links $j k$ and $j h$, where $j \in M_{j}$ is essential and $k$ and $h$ are two members of the $M_{j}$-group. Next, it is shown that for any $c$, this structure can be sustained in a PNE network if the component is sufficiently large. First, notice that $j$ gains intermediation rents from connecting the $M_{j}$-group to the rest of the component. The deletion of $j k$ (or $j h$ ) implies that this intermediation rent would have to be shared with another agent. Thus, for any $c$, there always exists a sufficiently large size of the component that makes the deletion of $j k$ (or $j h$ ) non-profitable. Second, if agent $k$ (or $h$ ) cuts the link $j k$ (or $j h$ ) off, then a new essential agent will intermediate the connection of the deviator with the rest of the component. Therefore, for any $c$, there always exists a sufficiently large size of the component that makes this deviation non-profitable. Finally, it is easy to see that $j$ does not have incentives to create a new link to this $M_{j}$-group, and no other member of $M_{j}$ will create a new link to the $M_{j}$-group whenever $c>\tilde{c}(t)$. 
Therefore, if $c>\tilde{c}(t)$ and the multi-department component is sufficiently large, then one essential member of the essential department $M_{j}$ should connect the $M_{j}$-group to the rest of the component.

Proof of Proposition 3. Let us assume that there is an essential agent $i$ in an extreme department, $M_{i}$. By Lemma 2, this department should have two links to another department, $M_{j}$, say $i j$ and $i k$. Agent $j$ 's marginal payoff for cutting her/his link off is $\Delta \Pi_{j}=c-\frac{m_{i}+1}{12}$, whereas agent $j$ 's marginal payoff for creating an additional link to $M_{i}$ is $\Delta \Pi_{j}=\frac{m_{i}-1}{6}-c$. These two marginal payoffs can be both negative only if $m_{i} \leq 3$.

On the other hand, let us assume that there is an essential agent $i$ in a non-extreme and non-essential department, $M_{i}$. The marginal payoff of a player $j$ for creating a new link that circumvents player $i$ to access the rest of members of $M_{i}$ is $\Delta \Pi_{j}=\frac{m_{i}-1}{6}-c$.

\section{References}

1. Burt, R.S. Structural Holes and Good Ideas. Am. J. Soc. 2004, 110, 349-399.

2. Mehra, A.; Kilduff, M.; Bass, D. The Social Networks of High and Low Self-Monitors: Implications for Workplace Performance. Adm. Sci. Q. 2003, 26, 121-146.

3. Podolny, J.; Baron, J. Resources and Relationships, Social Networks and Mobility in the Work Place. Am. Soc. Rev. 1997, 62, 673-693.

4. Ahuja, G. Collaboration Networks, Structural Holes, and Innovation: A Longitudinal Study. Adm. Sci. Q. 2000, 45, 425-455.

5. Burt, R.S. Brokerage and Closure: An Introduction to Social Capital; Oxford University Press: Oxford, UK, 2005.

6. Buskens, V.; van de Rijt, A. Dynamics of Networks if Everyone Strives for Structural Holes. Am. J. Soc. 2008, $114,371-407$.

7. Burt, R.S. Structural Holes: The Social Structure of Competition; Harvard Universtity Press: Cambridge, UK, 1992.

8. Bala, V.; Goyal, S. A Noncooperative Model of Network Formation. Econometrica 2000, 68, 1181-1230.

9. Goyal, S.; Vega-Redondo, F. Structural Holes in Social Networks. J. Econ. Theory 2007, 137, 460-492.

10. Kleinberg, J.; Suri, S.; Tardos, E.; Wexler, T. Strategic Network Formation with Structural Holes. In Proceedings of the 9th ACM Conference on Electronic Commerce, Chicago, IL, USA, 8-12 July 2008.

11. Haller, H. Network extension. Math. Soc. Sci. 2012, 64, 166-172.

12. Caulier, J.-F.; Mauleon, A.; Vannetelbosch, V. Contractually stable networks. Int. J. Game Theory 2013, 42, 483-499.

13. Jackson, M.O.; Wolinsky, A. A strategic model of social and economic networks. J. Econ. Theory 1996, 71, 44-74.

14. Dutta, B.; Mutuswami, S. Stable networks. J. Econ. Theory 1997, 76, 322-344.

15. Jackson, M.O.; van den Nouweland, A. Strongly stable networks. Games Econ. Behav. 2005, 51, 420-444.

(C) 2017 by the author. Licensee MDPI, Basel, Switzerland. This article is an open access article distributed under the terms and conditions of the Creative Commons Attribution (CC BY) license (http://creativecommons.org/licenses/by/4.0/). 